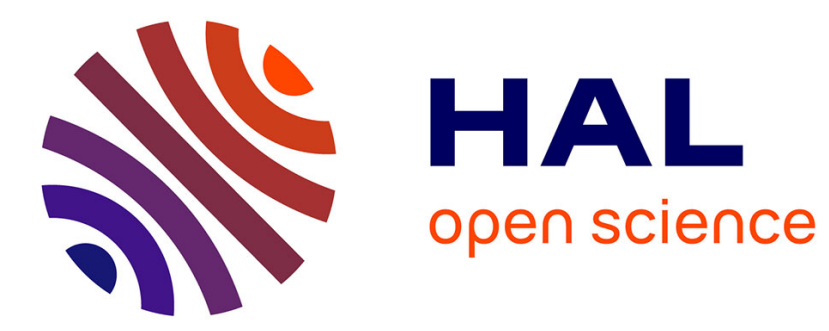

\title{
Langmuir Probe Diagnostics for Medium Pressure and Magnetised Low-Temperature Plasma
}

\author{
M. Tichy, P. Kudrna, J. Behnke, C. Csambal, S. Klagge
}

\section{To cite this version:}

M. Tichy, P. Kudrna, J. Behnke, C. Csambal, S. Klagge. Langmuir Probe Diagnostics for Medium Pressure and Magnetised Low-Temperature Plasma. Journal de Physique IV Proceedings, 1997, 07 (C4), pp.C4-397-C4-411. 10.1051/jp4:1997432 . jpa-00255588

\section{HAL Id: jpa-00255588 https://hal.science/jpa-00255588}

Submitted on 1 Jan 1997

HAL is a multi-disciplinary open access archive for the deposit and dissemination of scientific research documents, whether they are published or not. The documents may come from teaching and research institutions in France or abroad, or from public or private research centers.
L'archive ouverte pluridisciplinaire HAL, est destinée au dépôt et à la diffusion de documents scientifiques de niveau recherche, publiés ou non, émanant des établissements d'enseignement et de recherche français ou étrangers, des laboratoires publics ou privés. 


\title{
Langmuir Probe Diagnostics for Medium Pressure and Magnetised Low-Temperature Plasma
}

\author{
M. Tichý, P. Kudrna, J.F. Behnke*, C. Csambal* and S. Klagge**
}

Department of Electronics and Vacuum Physics, Faculty of Mathematics and Physics, Charles University, V. Holešovickách 2, 18000 Prague 8, Czech Republic

* Institute of Physics, Faculty of Mathematics and Natural Sciences, Ernst-Moritz-Arndt University, Domstrasse 10a, 17487 Greifswald; Germany

** Rosenweg 7, 17489 Greifswald, Germany

\begin{abstract}
In this contribution we aim at presenting the overview of the work that has been done in expanding the applicability of the probe method to low-temperature plasma at the pressure range when the collisions of charged particles with neutrals start to be important (we call this pressure range medium pressures) and to plasma under the influence of the weak-to-medium magnetic field that is commonly used in plasma enhanced technologies. Most of the discussion is devoted to simple case of a plasma consisting of electrons and of one kind of positive ions. Our review also mostly concerns the cylindrical Langmuir probe. The first part of the article is devoted to discussion on the influence of the positive-ion-neutral collisions on the interpretation of the ion current part of the probe characteristic in order to get the true value of the plasma number density. In the second part one of the theories that take account of this effect is used to assess the influence of the weak magnetic field to the interpretation of the probe data. Finally we discuss the anisotropy of the electron velocity distribution function due to the effect of the magnetic field. The discussion is supported by the new experimental data.
\end{abstract}

\section{INTRODUCTION}

Langmuir and Mott-Smith [1-5] firstly showed that it is possible to analyse properly the current-voltage probe characteristic in order to deduce plasma parameters in the vicinity of the probe. In particular, that it is possible to determine the plasma potential, the electron and ion densities and the electron energy distribution function. Assuming that the energy distribution of electrons is Maxwellian it is possible to determine the electron temperature too.

There are several potentially serious sources of errors in measurement of the probe characteristics in a DC plasma without the magnetic field. Some of them are coupled with the processing of the signal from the probe, some are effects that are coupled with the interaction of the probe and the plasma being investigated. Of the "apparatus" effects is worthy to mention the magnitude of the resistance in the probe circuit that should be much lower than the lowest differential resistance of the probe characteristic; the effect has been discussed in [6]. Among the second group we can list (i) too high probe collection area [7-9], (ii) the change of the work function over the probe surface (especially due to contamination of the probe surface) [10], (iii) the secondary emission of electrons from the probe surface, e.g. [11] and (iv) the collisions of charged particles with neutrals within the space charge sheath. If a magnetic field is applied to a plasma, then, in addition, (v) the orientation of the probe to the direction of the magnetic field is of prior importance. The effects (i,ii,v) cause "rounding off" the probe characteristic near the plasma potential and subsequently uncertainty in determination of the plasma potential and electron energy distribution function (EEDF). The effects (iii,iv) are at low-to-medium pressures mostly important in the positive-ion-current part of the probe characteristic and can cause changes of the measured value of the floating potential and of the estimated value of plasma number density. Our review concentrates on the effect of collisions and on the effect of magnetic field with regard to their influence of the probe data interpretation.

Article published online by EDP Sciences and available at http://dx.doi.org/10.1051/jp4:1997432 
The working regime of the probe in a plasma without the magnetic field is usually described by two parameters (see e.g. [12]) namely by the Knudsen number for ions and electrons $K_{i, e}=\lambda_{i, e} / R_{p}$ (where $\lambda_{i, e}$ is the mean free path for ions/electrons and $R_{p}$ the probe radius) and by the Debye number $D_{\lambda}=R_{p} / \lambda_{D}$, $\lambda_{D}=\left(\varepsilon_{o} k T_{e}\left(q_{o}^{2} n_{e}\right)\right)^{1 / 2}$ is the Debye length, $\varepsilon_{o}$ is the permittivity of vacuum, $k$ the Boltzmann constant, $T_{e}$ the electron temperature, $q_{o}$ the elementary charge and $n_{e}$ the electron density). Another parameter which influences the collection of charged particles by a probe is the "anisothermicity parameter" of a plasma namely the ratio of the electron to ion temperature, $\tau=T_{e} / T_{i}$. The introduction of this parameter (and also of $\lambda_{D}$ ) already implies the Maxwellian distribution of electron and (for the case $T_{i}>0$ ) of ion energies. The Maxwellian distribution of electron and ion energies is assumed also throughout the present paper except where stated otherwise. We restrict ourselves further to the case of a cylindrical probe which is easy to manufacture and is applicable in all cases where the distribution of electron and ion energies can be assumed isotropic. We also assume anisothermic plasma for which $\tau>>1$.

For most experimental conditions relevant to plasma-aided technologies the thickness of the probe sheath is proportional to the Debye length. Because $K_{i, e}$ characterises the magnitude of the ion and electron mean free path the quantity $\left(D_{\lambda} \cdot K_{i, e}\right)^{-1}$ is proportional to the number of collisions of these particles with neutrals in the sheath. Since the thickness of the sheath depends on the probe potential the proportionality coefficient is a function of the

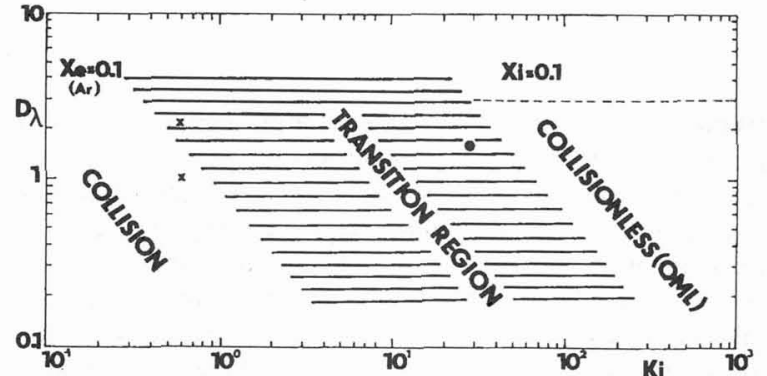

Figure 1. The division of the $D_{\lambda}-K_{i}$ plane to the collisionless, transition and collision probe regimes calculated for $\eta=15$ [12]. Working gas argon. The horizontal dashed line indicates the boundary of OML case [22]. $x \ldots$ data probe potential too. For a given probe from [60] taken at $62 \mathrm{~Pa}, \bullet .$. datum from [61] taken at $7.5 \mathrm{~Pa}$. potential the number of collisions of electrons and ions in the sheath can be visualised by means of the $D_{\lambda}$ vs $K_{i}$ plot. Such graph for $D_{\lambda} \leq 3$ is depicted in Fig. 1 . Similar plot has been introduced in [12] and [13]. From the graph in Fig. 1 it is seen that the working regime of the probe could be roughly divided into three regions separated by the lines depicting the number of collisions of ions/electrons with neutrals in the probe sheath for which are $X_{i, e} \cong 0.1$. The reasoning behind it is a simple consideration that in such case one can expect that the relative error in the measured ion or electron current due to collisions in the space charge sheath does not exceed $10 \%$, which is an acceptable error for most probe measurements.

In the probe working regime where $X_{i, e} \leq 0.1$ the influence of ion and electron collisions in the space charge sheath around the probe can be neglected. In this case the ion and electron probe currents can be described by a theoretical model that does not take the collisions between charged and neutral particles into account (shortly "collisionless model"). In case of $X_{i} \geq 0.1$ and $X_{e} \leq 0.1$ the electron probe current can be described by the collisionless model while in the ion current model the collisions in the space charge sheath should be taken into account. In the last case where both $X_{i}$ and $X_{e}$ are higher than 0.1 the collisions of both electrons and ions have to be taken into account in the probe current model.

The probe measurements in the medium pressure discharge correspond mostly to the transition and collision regimes. For this reason in order to analyse properly the current-voltage probe characteristics it is necessary to have the models of the probe current for these two regimes. The model for transition pressure regime is based on the collisionless model. In this model the electron current corresponds to the collisionless one and the positive ion current is corrected with respect to the collisionless one by the factor which expresses the influence of ion collisions in the space charge sheath around the probe.

In a magnetic field further parameter $\beta$ characterises the magnetic field strength with respect to the probe dimension, $\beta=r_{J} / R_{p}$, where $\mathrm{r}_{\mathrm{L}}$ is the mean Larmor radius that is for charged particles with a Maxwellian EEDF given by $r_{L}=\frac{1}{e B} \sqrt{\frac{\pi m_{e, t} k T_{e, i}}{2}}$. We do not have enough space in this report to describe in detail "classical" probe theories that analyse the collection of charged particles under the ideal, 
i.e. collision-free conditions; the reader should refer either to relevant books [9,11-15], or to original journal publications. For the case of so-called OML regime of the Langmuir probe that is most relevant for plasmas commonly used in plasma-aided technologies, i.e. when the sheath thickness is much larger than the probe radius, we would recommend the sources [3,16-20] and for the double probe method [21].

\section{LANGMUIR PROBE IN THE TRANSITION REGIME OF PRESSURES}

The collisionless OML model has been found satisfactory by experimenters for the electron collection by the probe for comparatively wide range of experimental conditions. However, the positive ion current was underestimated in this model that leads to overestimation of the positive ion density with respect to the electron one when determined from the same probe characteristic even at conditions when the plasma could be assumed as quasineutral. This has been found in non-isothermal discharge plasma, see e.g. [25] as well as in isothermal afterglow plasma [26]. Moreover the Bernstein-Rabinowitz [21] and Laframboise [22] theories predict that the ion saturation current should be for the orbital motion limited conditions (OML) independent of the Debye number $D_{\lambda}$ if the probe potential is constant. The calculated dependence of the ion current for the probe potential $\eta=\left(\eta_{F}+10\right)$ ( $\eta$ is the probe, $\eta_{F}$ the floating potential normalised to the electron temperature in $\mathrm{eV}$ ) is shown in Fig. 2 together with the curve presented by Chen [20] on the basis of Allen et al. theory [19]. In Fig. 2 the theoretical curves are compared with the experimental results presented in [27] and [28]. A comparison of the theoretical and experimental results has shown that in contrary to the Bernstein-Rabinowitz [21] and Laframboise [22] theories the ion saturation current depends on the Debye number $D_{\lambda}$ at orbital motion limited (OML) conditions and that its magnitude is larger than that predicted theoretically.

Experimental results obtained by several authors $[26,29,30]$ have been summarised by Smith and Plumb [26].

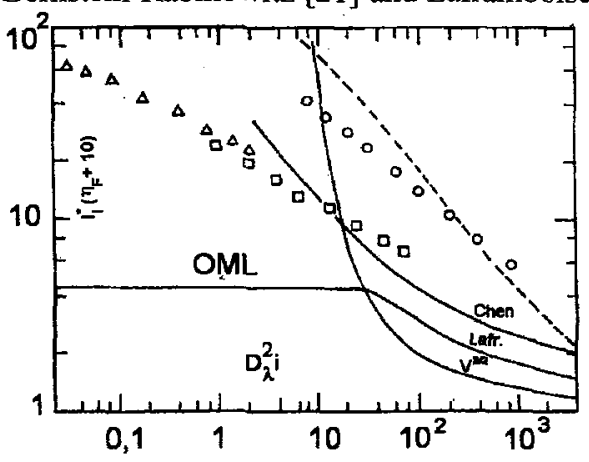

Figure 2. Comparison of the different collision-freetheories with experiment. -..-- spherical probe theory $[20,22]$, - cylindrical probe theories: Chen [20], Laft. [22], $\mathrm{V}^{3 / 2}$ [60]. Experiment: $\bigcirc R_{p}=1 \mathrm{~mm}, \Delta R_{p}=37,5 \mu \mathrm{m}$, $\mathrm{l}_{\mathrm{p}}=7 \mathrm{~mm}, \square R_{p}=0,375 \mathrm{~mm}$, measured in the positive column of $\mathrm{He}$ glow discharge at $25 \mathrm{~Pa}$. Current normalized with $n_{e}$ determined from the electron probe current at the plasma potential. From [27].

They have shown that the ion density $n_{i}$ determined from the ion current $I_{i}$ may be expressed in terms of the electron density $n_{e}$ determined from the electron current $I_{e}$ by the empirical relationship of the form $n_{i} \approx\left(1+0.07 \sqrt{m_{i}}\right) n_{e}\left(\left[\mathrm{~m}_{\mathrm{i}}\right]=\right.$ a.m.u. $)$. From the quasineutrality of the plasma follows, however, that the electron density $n_{e}$ should be equal to the positive ion density $n_{i}$ assuming that no negative ions are present within the plasma. The discrepancy between $n_{i}$ and $n_{e}$ has been observed in [26] even in the flowing afterglow plasma where the condition for the stable space charge sheath $T_{i}>(1 / 2) T_{e}$ (see e.g. $[14]$ ) is satisfied. The experimental results obtained in $[26,29,30]$ indicate therefore an inaccuracy in the assumptions of the OML model of the positive ion collection for the used experimental conditions. The discrepancy of $n_{i}$ and $n_{e}$ determination cannot lead back to an end-effect phenomenon (transition to spherical-like sheath configuration) [31].

We believe that these discrepancies can be, at least at certain simple experimental conditions, explained if the collisions of positive ions with neutrals in the space charge sheath around the probe are taken into account. Monte Carlo simulations [32] have shown, that already a small number of collisions in the sheath can destroy the orbital motion. The next paragraph is therefore devoted to discussion of probe theories that take account of the ion-neutral collisions in the sheath around the Langmuir probe.

\subsection{The collection of positive ions by the probe under the influence of ion-neutral collisions}

This effect has already been studied for many years; examples of earlier papers on this subject are [33-39]. The theories that treat the problem of the positive-ion collection by a Langmuir probe under the influence 
of collisions of ions with neutrals in the probe sheath can be divided into kinetic and continuum theories; we shall call such theoretical models "collision models" or "collision theories". The kinetic theories aim at description of the influence of the individual collision processes to the ion collection; the continuum theories use the hydrodynamical description. The kinetic approach can be regarded as more general since it describes the collisionless conditions too while the continuum theories require the supposition of many collisions. The kinetic theories discussed in this review present the effect of collisions in form of a correction to a collisionless ion collection model. Two collisionless models are used: Laframboise's [22] model that includes the classical Langmuir's orbital motion limited model (OML) [3] and the radial motion cold ion approximation model, so-called ABR-Chen $[19,20]$. We shall not present the thin sheath collision models such as "continuum plus free fall theory" [41,42] since they require higher plasma density than what is usually used in plasma-aided technologies.

The collision probe theory of the positive ion as well as of electron current to a cylindrical (spherical) probe has been presented by Chou, Talbot and Willis $[43,44]$ and describes the Langmuir probe characteristics at an arbitrary Knudsen number $0 \leq K_{i} \leq \infty$. The result of this theory is a reduction of the ion as well as of the electron probe current due to elastic scattering of these particles caused by collisions with neutrals in the probe sheath. The influence of collisions is calculated as correction to Laframboise's collisionless ion current $I_{i}^{*}$. This corrections was in a form more suitable for practical calculations presented by Talbot and Chou [43] and by Kirchhoff, Peterson and Talbot [45]. Similar as in Laframboise theory in the Talbot et al. theory $[43,44]$ the ion current is normalised using the factor $I^{*}$ so that $I_{i}^{*}=I_{i} / I^{*}$, where $I^{*}=A_{p} \sqrt{\frac{k T_{e}}{2 \pi m_{i}}} n q_{o}$ with $m_{i}$ being the mass of the ion and $A_{p}$ the surface collecting area of the probe. The correction factor $F_{T}$ which expresses the reduction of the ion current due to the elastic scattering can be written according to the theory by Talbot and Chou [43] as $F_{T}=\frac{I_{i}^{*} L}{I_{i}^{*}}=1+\frac{\tau^{1 / 2} J_{i} I_{i}^{* L}}{K_{i}}$. In these expressions $J_{i}=\int_{0}^{1} \frac{\exp (-\tau \eta)}{Z} d Z, I_{i}{ }^{{ }_{L}}$ and $I_{i}^{*}$ are the normalised ion currents in the collisionless and collision case respectively, $\eta$ is the probe potential normalised to electron temperature in $\mathrm{eV}, Z$ is the reciprocal of the radial distance from the probe $r$, normalised to the probe radius $R_{p}, Z=\left(R_{p} / r\right)$. Talbot and Chou [43] proposed to determine the integral $J_{i}$ at an arbitrary value of $K_{i}$ using an ad hoc interpolation formula $J_{i}=J_{i \infty}+\left(J_{i 0}-J_{i \infty}\right)\left(1+K_{i}\right)^{-1}$, where $J_{i \infty}$ and $J_{i 0}$ are the integrals $J_{i}$ calculated at the collisionless and continuum limit respectively. The calculation procedure of these integrals is described in detail in [43], therefore we limit ourselves here only to the comments concerning the applicability of this procedure in the OML conditions. In the calculations of the collisionless limit of this integral, $J_{i \infty}$, the authors use the Bernstein and Rabinowitz theory [21] which assumes the monoenergetic ion distribution. In case of a cylindrical probe Laframboise's calculations [22] showed that the ion current calculated assuming Maxwellian and monoenergetic ion energy distribution differ very little from each other in the OML regime $\left(D_{\lambda}<3\right)$. This fact supports the applicability of the procedure calculating the integral $J_{i \infty}$ described in [43] in case of a cylindrical probe also in the OML regime. Since the calculation of the continuum limit of the integral $J_{i 0}$ does not present any problems concerning the applicability at OML conditions the overall conclusions is that for cylindrical probe the approximate Talbot and Chou procedure [43] can be applied in the OML regime.

It is to be noted that there were also other kinetic models for the description of the charged particle collection by a Langmuir probe. Bienkowski and Chang [46] found the solution of Poisson and Boltzmann equation with the collision term for the limiting case $D_{\lambda} \rightarrow \infty$ while Wasserstrom et al. [47] only in the limiting case of $\eta \rightarrow 0$. Both limits can be, however, derived from the Talbot and Chou theory with the same results. Substantially simpler than the Talbot and Chou theory is the procedure employed by Self and Shih [48] that modified the ABR radial model (for spherical probe) [19] by introduction of the "friction term". The results are presented as dependencies of the normalised ion current at a certain probe 
voltage on the $D_{\lambda}$ and the ion-neutral collision frequency $v_{i n}$. The same procedure is applied for cylindrical probe to the ABRChen $[19,20]$ radial model in [49].

Klagge and Tichý in the paper [27] carried out a set of numerical calculations of the positive ion current as a function of the Knudsen number $K_{i}$ at the normalised potential $\eta=\eta_{F}+10$ based on the procedure [43]. The difference from the original Talbot and Chou paper [43] consisted in the fact that they used the ABR-Chen $[19,20]$ collisionless current $I_{i}{ }^{* A}$ in place of $I_{i}{ }^{2} L$ in $F_{T}$ calculation. The reason for this was that this model $\left(I_{i}^{*}{ }^{*}\right)$ with a reasonable accuracy described the experimental results at conditions close to collision-free. The authors used their own analytical approximation for the numerical Chen [20] currents. This approximation has been presented in [48] and can be written as $I_{i}^{* A}\left(\eta_{w}\right)=a\left(\eta_{w} / b\right)^{\alpha}$, where $a=\left(D_{\lambda}+0.6\right)^{0.05}+0.04$, $b=0.09\left[\exp \left(-D_{\lambda}{ }^{-1}\right)+0.08\right]$ and $\alpha=\left(D_{\lambda}+3.1\right)^{-0.6}$. At a given $\eta_{w}$ (so-called working point, usually $\eta_{w}=\eta_{F}+10$ or $\eta_{w}=15$ is selected for the calculation) this formula presents the dependence of the $I_{i}^{* A}$ on $D_{\lambda}$, or in turn, on $D_{\lambda}{ }^{2} I_{i}{ }^{* A}$ in accordance with [20] with a fairly negligible error. The parameter $D_{\lambda}{ }^{2} I_{i}^{*}$ has the advantage that it does not depend on the electron density and can be therefore directly evaluated from the experimental data using the experimentally determined electron temperature. The dependence $I_{i}^{*}$ vs $D_{\lambda}{ }^{2} I_{i}^{*}$ is generally called Sonin-plot [23]. The results of the corrections presented in [27] are shown in Fig. 3. From this figure it is possible to determine the correction factor $F_{T}$ by means of which the experimental value of the ion probe current can be corrected with respect to collisions. The exact step-by-step procedure described in [27] enables to set-up a program that calculates this correction directly without the necessity to analytically approximate.the data in Fig. 3.

Zakrzewski and Kopiczynski [51] have introduced the other model in which elastic collisions of ions with neutral particles have on the contrary to Talbot and Chou theory two consequences: the destruction of the orbital motion of ions and the elastic scattering of positive ions. As compared to the Talbot and Chou theory the destruction of the orbital motion can lead to an increase of the positive ion current. The effect of the orbital motion destruction predominates for lower pressures when the mean free path of positive ions is greater or comparable to the sheath thickness. The elastic scattering of the positive ions causes a monotonous decrease of the positive ion current and dominates for higher pressures. As a result the current peak appears at a pressure when approximately one collision of the ion with the neutral in the space charge sheath occurs. The resulting normalised dimensionless ion current $I_{i}^{*}$ to a cylindrical probe is then $I_{i}^{*}=\gamma_{1} \gamma_{2} I_{i}^{* L}$, where $\gamma_{l}$ describes the rate of increase of ion current due to destruction of orbital motion and $\gamma_{2}$ corresponds to the rate of reduction of ion due to scattering, $I_{i}{ }^{*} L$ is the normalised collisionless ion probe current predicted by Laframboise [22]. The correction factor $F_{Z}$ is given as $F_{Z}=\frac{1}{\gamma_{1} \gamma_{2}}$. Zakrzewski and Kopiczynski have derived the coefficient $\gamma_{I}$ under assumptions that at the orbital motion limited conditions the positive ion current collected by a perfectly absorbing probe is saturated and it is described by Laframboise theory. This physical argument can be expressed analytically. They assumed that the dimensionless ion current at the sheath edge $I_{i}^{* A}$ is given approximately by Allen et al. theory [19]. In the collisionless limit only the current $I_{i}{ }^{*} L$ predicted by Laframboise reaches the probe surface. The current $\left(I_{i}^{*}{ }^{* A}-I_{i}{ }^{*} L\right)$ leaves the sheath due to the orbital motion.

When an orbiting positive ion undergoes a collision with neutral particle in the space charge sheath it looses energy and is attracted to the probe. An average ion makes $X_{i}=S / \lambda_{i}$ collisions in the sheath if we denote by $S$ the thickness of the sheath and by $\lambda_{i}$ the ion mean free path. According to Zakrzewski and Kopiczynski the rate of increase of the positive ion current due to the destruction of the orbital motion by 
elastic collision is $\gamma_{1}=1+\left(\frac{l_{i}^{* A}}{I_{i}^{* L}}-1\right) \frac{S}{\lambda_{i}}$. This equation is valid for $S / \lambda_{i}<1$. For $S / \lambda_{i} \geq 1$ total destruction of the ion orbital motion occurs and $\gamma_{1}=\frac{I_{i}^{* A}}{I_{i}^{*}}$. The average amount of the ion collisions with the neutral particles within the sheath equals roughly $X_{i}=S / \lambda_{i}$. Kopiczynski [52] determined the thickness of the sheath $S$ on the basis of numerical calculations carried out by Basu and Sen [53]. Then $X_{i}=\frac{S}{\lambda_{i}}=\frac{\sqrt{\bar{\eta}}}{D_{\lambda} K_{i}}$, where $\bar{\eta}=m(\eta+3.5)-4$ and $m \cong 0.59+1.86\left(D_{\lambda}\right)^{0.47}$. The corresponding reduction rate of the ion current due to the elastic scattering $\gamma_{2}$ Zakrzewski and Kopiczynski estimated according to Schulz and Brown [54] and Jakubowski [55]. It should be noticed that for a given probe potential $\gamma_{1}$ and $\gamma_{2}$ are function of both $R_{p} / \lambda_{D}$ and $\lambda_{i} / R_{p}$

For normalised probe potential $\eta_{w}=15$ Kopiczynski [52] carried out extensive numerical calculation of the dependence of the quantity $I_{i}^{*} D_{\lambda}{ }^{2}$ on the ion Knudsen number $K_{i}$ with the Debye number $D_{\lambda}$ as a parameter. David [56] and David et al. [57] extended these calculations towards lower Knudsen numbers $K_{i}$ occurring in a medium pressure discharge. The comparison of the already mentioned theories of the ion current collection by the probe in the transition regime has been made by David [56]. The results of his calculations are depicted in Fig. 4.

It can be deduced from this figure that the results of Talbot and Chou and those of Zakrzewski and Kopiczynski are not in contradiction within the region $2 \leq D_{\lambda} \leq 3$ since the ion current maximum predicted by Zakrzewski and Kopiczynski is within this region very flat and the results of both theories differ only a little from each other. For lower $D_{\lambda}\left(D_{\lambda}<2\right)$ the ion current calculated by using the Zakrzewski and Kopiczynski theory $[51,52]$ exhibits a well pronounced maximum at $X_{i} \cong 1$. At higher values of $X_{i}>1$ the ion current decreases with decreasing $K_{i}$ more rapidly in comparison with the ion current obtained from theory $[43,44]$ in which no current peak is observed.

Since the Chou and Talbot theory does not take into account the effect of the probe current increase due to the orbital motion destruction caused by ion collisions with neutrals within the probe sheath, the

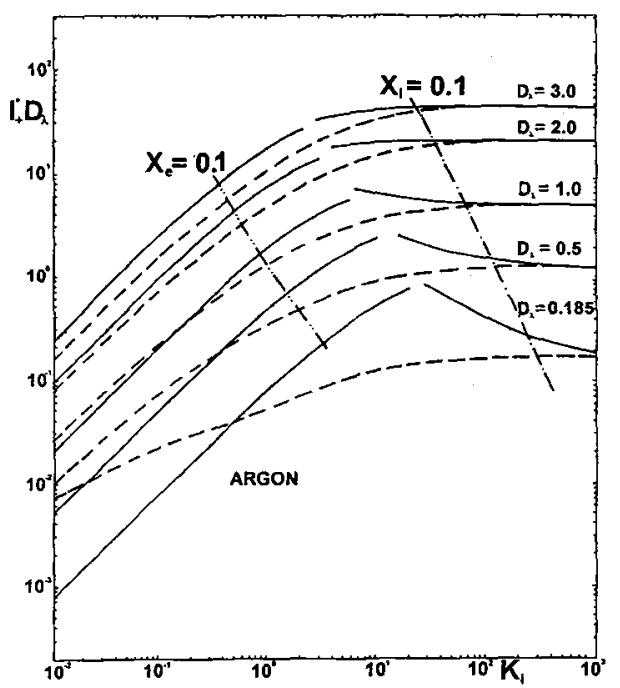

Figure 4. The dependence of the directiy measurable quantity $I_{i}^{*} D_{\lambda}^{2}$ on $K_{i}$ with $D_{\lambda}$ as a parameter calculated in [56] using the theory [51] (full line) and the theory [43,44] (broken line). The chain lines indicate the boundaries of the transition probe regime in the same way as in Fig. 1. theory developed by Zakrzewski and Kopiczynski describes at OML conditions the ion collection by a Langmuir probe in the region where not all the ions suffer a collision with a neutral particle within the probe sheath $\left(X_{i}<1\right)$ more precisely than the Talbot and Chou theory. On the other side for larger number of ion collisions within the sheath $\left(X_{i}>>1\right)$ the theory developed by Zakrzewski and Kopiczynski is not applicable at OML conditions since it uses for the evaluation of the factor $F_{Z}$ the formulae which have been derived in [55] under the assumption of only a few ion collisions within the probe sheath. In order to extend the validity of the theory developed by Zakrzewski and Kopiczynski [51,52] for an arbitrary $K_{i}$ we suggested in [12] to apply the Talbot and Chou theory $[43,44]$ for determination of the factor (or its equivalent) describing the effect of scattering of ions due to their collisions with neutrals within the probe sheath. The corresponding expression following the model used by Zakrzewski and Kopiczynski (see eq.(5)) is $I_{i}^{*}=\gamma_{1} \gamma_{2}^{*} I_{i}^{*} L$, where $\gamma_{2}^{*}$ is the coefficient describing the effect of the ions scattering due to collisions with neutrals within the probe sheath determined from Talbot 
and Chou model [43]. The advantage of the new model [12] of the ion collection by a cylindrical Langmuir probe consists mainly in the fact that it is valid for any $K_{i}$ i.e. for $0 \leq K_{i}<\infty$ as long as the condition of the OML regime of the probe is fulfilled $\left(D_{\lambda} \leq 3\right)$. On the contrary to the calculations of the $\gamma_{2}$ factor which have been made by Zakrzewski and Kopiczynski the correction factor $\gamma_{2}{ }^{*}$ depends on the ratio of the electron to ion temperature $\tau=\left(T_{e} / T_{i}\right)$. We suggested in [12] to call this new model the "modified Talbot and Chou model" since it refines the "classical" kinetic Chou and Talbot theory [43,44].

Comparison of the theory [12] with the Chen-Talbot [27] and with the Zakrzewski-Kopiczynski $[51,52]$ theories has been made by Kudrna in [58]. He calculated also the dependence of the directly measurable quantity $D_{\lambda}^{2} I_{i}^{*}$ on $K_{i}$ with $D_{\lambda}$ as a parameter. Samples of his results are presented in Fig. $5 \mathrm{ab}$.

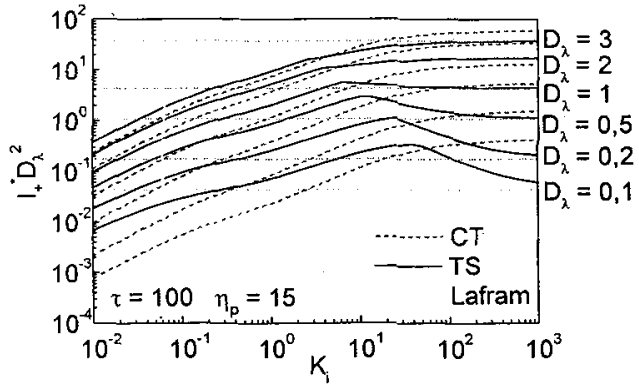

Figure 5a. Comparison of the Chen-Talbot positive ion current model [27] with modified Chou-Talbot model [12]. For comparison also collisionless current by Laframboise [22] is shown.

Note that in both figures the ion current calculated according [12] decreases at lower $K_{i}$ with decreasing $K_{i}$ slower than that from both theories [27] and [51,52].

\subsection{Comparison of "collision theories" of positive ion collection by the probe with experiment}

Up to now the Zakrzewski and Kopiczynski model [51] has been tested in several papers [12,52,59,60]. In $[12,52]$ the authors used the X-band microwave interferometer for the independent determination of the relative values of the electron density. The double probe technique was used for the determination of the electron temperature. A set of single cylindrical probes was applied to obtain the probe characteristics. The reasonable quantitative agreement supports the model of ion collection carried out by Zakrzewski and Kopiczynski in the region of $X_{i}<1$. For support of this theory speak also results of the work [60] obtained in an argon afterglow discharge at the pressure $62 \mathrm{~Pa}$ and of [61] measured in an RF argon discharge at the pressures around $10 \mathrm{~Pa}$.

Thorough assessment of the applicability of all mentioned collision theories has been presented in [59]. The measurements have been done in a low pressure flowing afterglow system with a Langmuir probe and downstream mass analysis (FALP). The experiments have been done in collaboration with the Innsbruck University where the FALP system was available as well as on the FALP system at the Charles University in Prague. Several kinds of ions have been

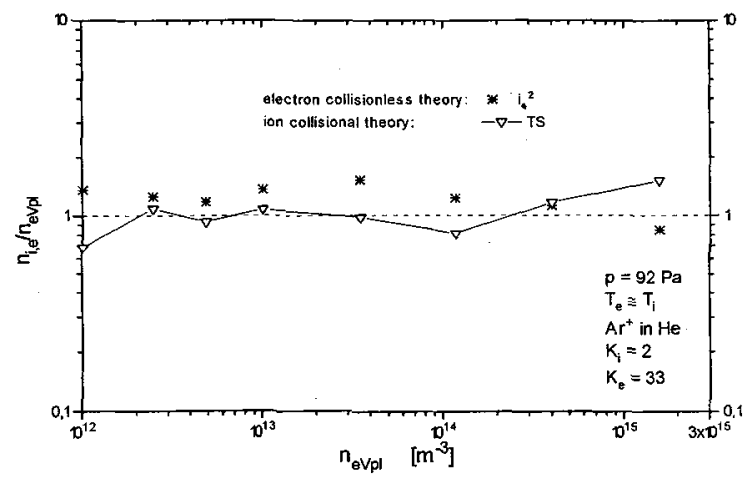

Figure 6. The comparison between the electron density calculated from the OML theory and the ion density calculated using theory [12] (denoted as TS) plotted as a ratio to the electron number density evaluated from the electron current at the space potential (also plotted on $x$-axis) at isothermal conditions. (From [59].) 
used for this study. Fig. 6 presents typical results that have been obtained with rare gas ions ( $\mathrm{Ar}^{+} \mathrm{and}_{\mathrm{Kr}}^{+}$) in the He carrier gas. The plot shows the numerical values of the ratio of ion density according the theory [12] to the electron density estimated from the electron current at the plasma potential vs the numerical value of the electron density estimated from the electron current at the plasma potential $\left(n_{e V p l}\right)$. In addition also the ratio of the electron density estimated from the $I_{e}^{2}$ vs $V_{p}$ plot $\left(n_{e l e s q}\right)$, see e.g. [26] to the $n_{e V p}$ is plotted on the ordinate axis (* points) in order to support the choice of the density value $n_{e V p l}$ as the reference one.

There is a steady effort to improve the understanding of the probe method in order to obtain more reliable data from the probe diagnostic [64-67]. The present state of knowledge on the influence of positive ion-neutral collisions in the probe sheath does not, however, allow to recommend the universally applicable theory that would give satisfactory results in a broad range of experimental conditions; for afterglow conditions the theory [12] yields fairly consistent results, for an active plasma (also when the supposition of the Maxwellian EEDF is not quite satisfied) the ABR-Chen theory with the collision correction [27] gives the best agreement with the experiment. More general results might bring the Monte-Carlo simulation of the ion motion in the probe vicinity; the first steps have been done in [32].

\section{LANGMUIR PROBE IN A MAGNETISED PLASMA}

The low-temperature-plasma-aided creation and etching of thin films are due to their broad applications possibilities very important parts of plasma-chemistry. Important drawback of many plasma enhanced surface treatments is the comparatively low grow/etch rate compared to classical chemical or electrochemical processes. In many technological applications of the low-temperature plasma to creation and etching of thin films is therefore used magnetic field that helps the plasma confinement and increases the grow/etch rate. The magnetic field in these systems can be either nonhomogeneous (created typically by permanent magnets; one example is the planar unbalanced magnetron) or almost homogeneous (created by coils) and its strength does not usually reach too high values. When using the Langmuir probe as a diagnostic tool in these systems the question arises up to what limit of the ratio $B / p$ (magnetic field strength to the working pressure; this is a similarity parameter in magnetised plasma) it is possible to use the conventional methods for evaluation of the basic plasma parameters such as charged particle density and electron energy distribution function (EEDF) from the Langmuir probe data.

Although the first discussions on the Langmuir probe saturated currents in a magnetic field have been published already at the end of forties $[15,18]$ the more intensive

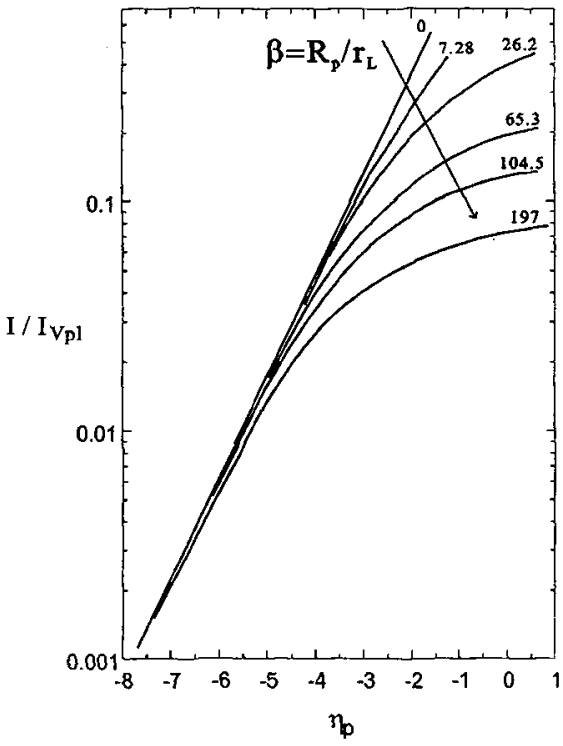

Figure 7. The influence of the magnetic field characterised by the parameter $\beta$ on the spherical probe electron current (normalised to that at the space potential $\left.\mathrm{V}_{\mathrm{p} 1}\right)$. From [74]. research on the use of the probe diagnostics in a low-temperature plasma in a magnetic field begun at the end of sixties [67-93] and proceeds up to the present time [11,94-99]. The decisive arguments have been given in the paragraph above, additional reasons are that the Langmuir probe is also often used to study the edge plasma in large magnetic fields in hot plasma devices such as tokamaks [100-107]. Not too many papers are devoted to the more complicated case of concerted action of higher pressures and magnetic field to the probe measurements [108-110] even if this case is from the viewpoint of technological applications the most interesting one. 
The discussion of the influence of the magnetic field to probe measurement in the collision-free case can be, in accord with [16] divided in 4 categories in dependence on the parameter $\beta=R_{p} / r_{L} .:$ (i) At $\beta<<1$, weak $B$, the influence is small and can be neglected (rare case of technologically interesting applications), (ii) at $\beta \approx 1$ the $B$ is still weak, but it is sometimes necessary to introduce small corrections (frequent case in plasma-aided technologies), (iii) at $\beta>1$ the $B$ is strong but it is still possible to interpret at least part of the Langmuir probe characteristic (case of tokamak edge plasma) and (iv) at $\beta>>1$, i.e. at very strong $B$ the probe characteristic is no longer interpretable. From the definition of the parameter $\beta$ it is evident that $\beta$ decreases with increasing charged particle velocity and mass; therefore the faster electrons and the ions are less influenced by the magnetic field during their collection by the probe. In other words, least distorted part of the probe characteristic due to the effect of magnetic field shall be that for very negative probe with respect to plasma, see e.g. $[74,77]$. The changes in the probe characteristic when the probe is applied in a magnetized plasma are therefore most apparent in the region close to the plasma potential. The directional movement of charged particles along the magnetic field lines reduces the diffusion of particles in direction across the field lines. If we consider the electron motion that is mostly influenced by the magnetic field then their diffusion coefficient across the field $D_{\perp}$ reduces to $D_{\perp}=D_{0} /\left(1+\Omega_{e}{ }^{2} \tau_{e n}{ }^{2}\right)$ with $D_{0}=v_{t h}^{2} \tau_{e n}$, where $\Omega_{e}=q_{0} B / m_{e}, \tau_{e n}=1 / v_{e n}$ and $v_{t h}$ are the electron cyclotron frequency, electron-neutral collision time and electron thermal velocity respectively [113]. In case of $\Omega_{e} \tau_{e n} \gg>1$ (magnetized plasma at low pressures) that is the case of most technologically used plasmas the expression for $D_{\perp}$ reduces to $D_{\perp} \approx v_{t h}{ }^{2} / \Omega_{e}{ }^{2} \tau_{e n}=r_{L}{ }^{2} / \tau_{e n}$. In other words in direction perpendicular to the magnetic field the effective mean free path of electrons is roughly equal to the Larmor radius. If the probe draws too much current at probe potentials close to the plasma potential the electrons are absorbed by the probe more rapidly than they can be supplied by diffusion from the distant regions where they are produced. Further effect concerns the change of the effective collecting area of the probe, since the charged particles flow to the probe mostly from the direction of the field lines reducing hence the original probe area to double of its projection to the field direction. Finally, at higher magnetic fields, e.g. in tokamaks, the probe is connected to its reference electrode only by the "current tube", that reduces the ratio of the reference-electrode-probe surface collecting areas. All three effect then lead to "blurring of the knee" of the probe characteristic near the space potential as it is seen in Fig. 7. When for the data analysis from such affected probe the conventional methods are used the resulting plasma number density is underestimated, plasma potential shifted towards probe retarding voltages and the electron temperature deduced from the slope in the electron retarding regime is overestimated (the slope is slower). Note that this effect does not depend on the magnetic field strength only, but rather on the ratio $\Omega_{e} / v_{e n}$, or on $B / p$. The degree of anisotropy of the problem (and hence of the influence to the probe measurements) will therefore depend also on $B / p$ and not on $B$ itself. The assessment of the error caused by the effect of magnetic field to the probe data and
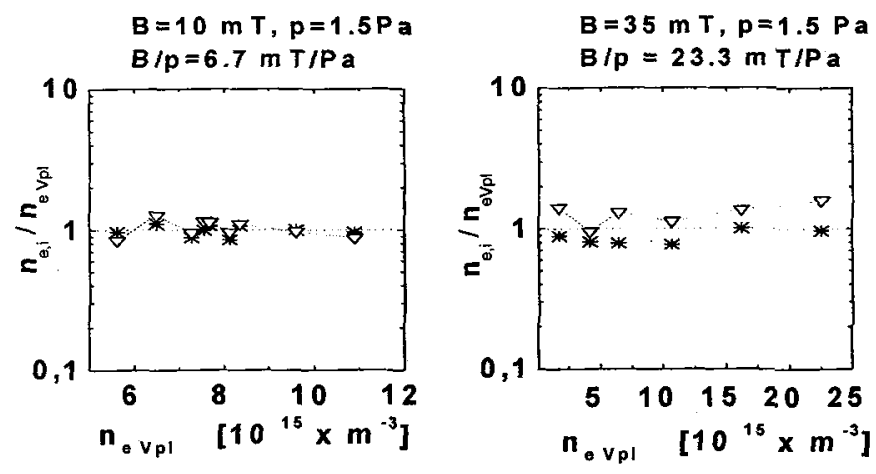

consequently to accuracy of estimated plasma parameters is therefore most interesting.

Recent experimental assessment of this effect at weak-to-medium magnetic fields has been presented in $[11,100]$. The work was based on the assumption that at the range of the pressure and the magnetic field employed the influence of the magnetic field on the positive ion collection by the probe is negligible. The

Figure 8. Comparison of the relative positive ion $(-\nabla-$, theory [27]) and electron densities $(*$, OML theory $[3,22])$ estimated from the same probe data at different magnetic fields. DC cylindrical magnetron discharge, working gas argon. Reference electron density from the electron current at plasma potential. From [95]. comparison of the positive ion density estimated from the ion accelerating region of the probe 
characteristic with the electron density estimated from the electron current at the plasma potential (by conventional method, i.e. without any correction to the magnetic field) at lower and higher $B / p$ should then give an idea of the magnitude of this influence. Sample of the results from [100] is given in Fig. 8. The figure is arranged in a similar manner as Fig. 6 . It is seen that while at the low $B / p$ there is an agreement between the estimated values of ion and electron density, at higher $B / p$ the electron density estimated from the electron current at the space potential is systematically underestimated; the difference gives a measure of the error due to the magnetic field.

Anisotropy of the electron temperature in a magnetic fields has been studied e.g. in [112-114]. Of those we shall briefly mention that by Aikawa [113]. He used two Langmuir probes for determination of the perpendicular and the parallel components of the electron temperature, what represents the first approximation of a direction-resolved probe diagnostic. Both components of the electron temperature, $T_{\|}$ and $T_{\perp}$ he obtained by the "conventional" method, i.e. from the slope of the semilogarithmic plot of the current drawn either by the planar probe whose surface was perpendicular to the $B$-field lines or by the cylindrical Langmuir probe whose axis was parallel to the $B$-field lines in the electron retarding regime respectively. His typical results taken at the argon pressure $1.6 .10^{-2} \mathrm{~Pa}$ are shown in Fig. $9(\Theta$ is the angle between the probe axis and the magnetic field lines). The electron temperature measured at the angle $\Theta$ exceeds up to several times the parallel one $T_{\| \mid}$. Aikawa further computed that depending on the ratio of the probe radius $R_{p}$ to the sheath radius $r_{s}$ the ratio of $T_{\perp} / T_{\mid l}$ can at the same $B$ and $p$ as in Fig. 9 exceed the value 10 (at $R_{p} / r_{s} \approx 0.1$, i.e. at large sheath radius compared to the probe one). It is to be noted, that the Aikawa-theories for cylindrical and plane probe are selfconsistent. Both yield the same values for space potential and plasma density, while the cylindrical probe indicates $T_{\perp}$ and the plane probe indicates $T_{\|}$.

The work [115] studied the radial distribution of the EEDF in the positive column of a glow discharge in neon in the magnetic field collinear with the electric field. The authors used the "conventional" second harmonic method to obtain the second derivative of the probe characteristic from which they calculated the electron density and the electron mean energy. They found that even at comparatively low values of $B / p$ of the order of $1.10^{-3} \mathrm{~T} / \mathrm{Pa}$ the magnetic field influences the radial distribution of the mean electron energy in the sense that higher $B / p$ causes the increase of the mean electron energy at larger radii.

The recent work by Arslanbekov et al. [116] discusses the EEDF measurements by a Langmuir probe at elevated pressures and in magnetic field. He creates an analogy between the effect of an increased pressure and the effect of the magnetic field on the probe characteristic. However in the analysis of the influence of the magnetic field on the

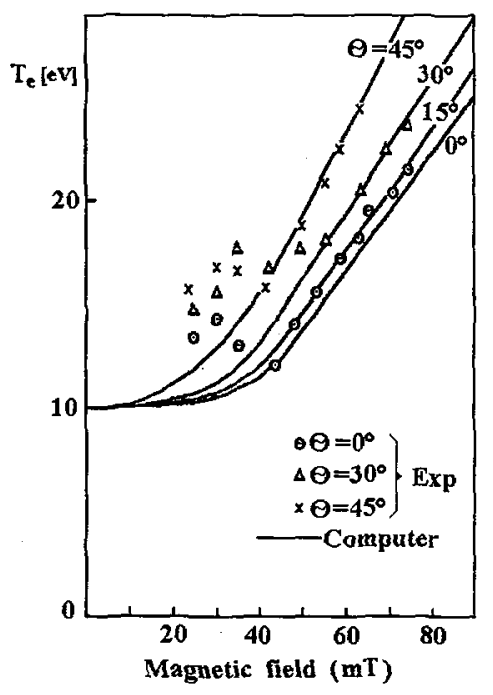

Figure 9. Computed (solid lines) and measured (points) $T_{\perp}$ by Aikawa [113] at $B / p \approx 3 \mathrm{~T} / \mathrm{Pa}$ and assumed $T_{\eta}=10 \mathrm{eV}$. cylindrical probe in a plasma the case of a thin sheath is assumed and hence this work is not directly applicable to the low temperature discharge plasma of technological interest since in such case the sheath thickness is usually large or comparable to the probe radius.

\section{EXPERIMENTAL ASSESSMENT OF THE ANISOTROPY OF THE EEDF IN A MAGNETIC FIELD}

Under the assumption that the EEDF is isotropic there exist many papers that are devoted to its probe studies in different experimental conditions, e.g. [116-118]. For the experimental study of the anisotropy of the EEDF the use of the method suggested by Mesentsev et al. [119-126] and by Klagge and Lunk 
[127] is proposed. This method consists in the expansion of the anisotropic electron velocity distribution function (EVDF) in a series of spherical (Legendre) polynomials

$$
f(v, \Theta)=\sum_{i=0}^{\infty} f_{i}(v) P_{i}(\cos \Theta) .
$$

Basic assumptions for the implementation of the procedure are:

- collisionless movement of the particles collected by the probe,

- negligibility of reflection and secondary emission of electrons from the probe,

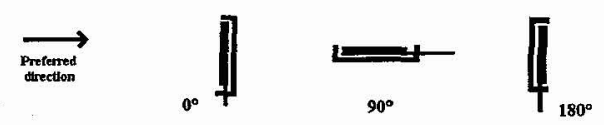

- cylindrical symmetry of the EVDF around the Figure 10. Possible orientation of the planar probe. preferred direction.

The coefficients in the expansion series are determined from the measurements by the planar Langmuir probe at different angles to the preferred direction, in this case to the direction of the magnetic field vector. Generally one obtains so many coefficients $f_{i}$ for the into Legendre polynomial expanded EVDF as probe position is used. The accuracy of the determination of the probe data does not usually allow to determine more than the first two or three coefficients of this series, the first one being the isotropic one the second one characterizing the drift and the third one characterizing the second order anisotropy. For determination of the first three coefficients we need the probe data measured in three directions, see Fig. 10.

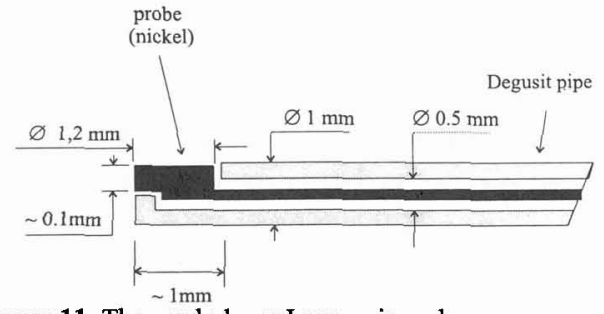

Figure 11. The used planar Langmuir probe.

From the probe data we get the required quantities as follows:

1. The electron density $n_{e}=\left(\frac{1}{3}\right) 2^{1 / 2} m_{e}^{1 / 2} e^{-3 / 2} S^{-1} \int_{0}^{\infty} V^{1 / 2}\left(I_{0}^{\prime \prime}+4 I_{90}^{\prime \prime}+I_{180}^{\prime \prime}\right) d V$

2. The isotropic part of the electron velocity distribution function (EVDF)

$$
F_{0}\left(\sqrt{2 e V / m_{e}}\right)=\left(\frac{2}{3}\right) e^{-2} m_{e} n_{e}^{-1} S^{-1} V\left(I_{0}^{\prime \prime}+4 I_{90}^{\prime \prime}+I_{180}^{\prime \prime}\right)
$$

or the isotropic part of EEDF

$$
f_{o}(e V)=\left(\frac{1}{3}\right) 2^{1 / 2} e^{-5 / 2} m_{e}^{1 / 2} n_{e}^{-1} S^{-1} V^{1 / 2}\left(I_{0}^{\prime \prime}+4 I_{90}^{\prime \prime}+I_{180}^{\prime \prime}\right)
$$

3.The first order anisotropy of the EEDF

$$
f_{1}(e V)=2^{1 / 2} e^{-5 / 2} m_{e}^{1 / 2} n_{e}^{-1} S^{-1} V^{1 / 2} g_{1}(e V), g_{1}(e V)=G_{1}+(2 e V)^{-1} \int_{e V}^{\infty} G_{1} d \varepsilon \text {, where } G_{1}=\left(I_{0}^{\prime \prime}-I_{180}^{\prime \prime}\right)
$$

4.The second order anisotropy of the EEDF $f_{2}(e V)=\left(\frac{2}{3}\right) 2^{1 / 2} e^{-5 / 2} m_{e}^{1 / 2} n_{e}^{-1} S^{-1} V^{1 / 2} g_{2}(e V)$, where

$$
g_{2}(e V)=G_{2}+\left(\frac{3}{2}\right)(e V)^{-3 / 2} \int_{e V}^{\infty} \varepsilon^{1 / 2} G_{2} d \varepsilon \quad, \quad G_{2}=\left(I_{0}^{\prime \prime}-2 I_{90}^{\prime \prime}+I_{180}^{\prime \prime}\right)
$$

For detailed description of the procedure with 5 probe orientations see e.g. [121, 127]. It is to be noted that the anisotropy of the EEDF in the magnetic field should be regarded in similar manner as the anisotropy of the electron temperature in [113], i.e. as the anisotropy of the electron velocity components. The expansion (1) requires the EVDF to be symmetrical around the preferred direction and hence cannot be used generally. In magneto-plasmas without drift the preferred directions $\vec{B}$ and $-\vec{B}$ are equivalent. However, the measurement at $\Theta=0$ and $\Theta=180^{\circ}$ are advantageous for discharge end-effect studies. In our case of the measurement in a positive column of a DC magnetron discharge, where the electric field is very small the poloidal asymmetry of the EEDF due to the $\vec{E} \times \vec{B}$ drift could be neglected. 
The experiment has been performed on the DC cylindrical magnetron discharge system in the Institute of Physics, University of Greifswald, Germany. The system differed from that described in [11] only by the used probe. In the presented measurements the probe was planar, see Fig. 11. The 3 orientations with regard to the vector of the magnetic field were adjusted by rotating the probe along its axis that was positioned perpendicular to magnetic field lines. Hence $f_{0}$ and $f_{2}$ were obtained in the expansion (1); $f_{l}$ and generally all odd terms should vanish, if the plasma anisotropy is produced only by the magnetic field, see Eq. (2). Because of discharge instabilities we could not operate with pressures lower than $0.3 \mathrm{~Pa}$.

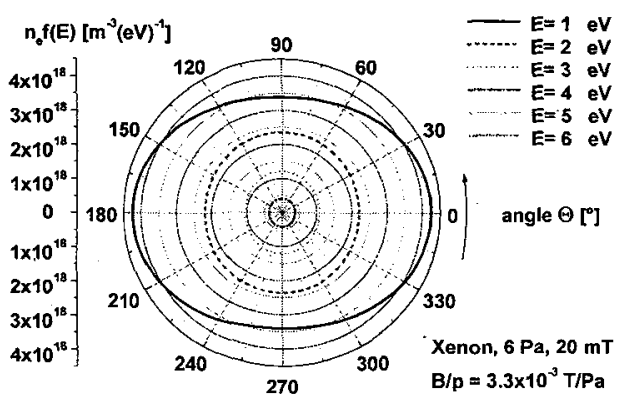

Figure 12. Polar plot of the EEDF in xenon at $6 \mathrm{~Pa}$ and $B=2.10^{-2} \mathrm{~T}$.

We present two sets of data, at higher and lower value of $B / p$. The results are presented as polar plots of the magnitude of the EEDF obtained from Eq. (1) at several different energies.

In Fig. 12 there is the EEDF at $B / p=3.3 \cdot 10^{-3} \mathrm{~T} / \mathrm{Pa}$. The data have been acquired in the cylindrical magnetron discharge in xenon at the pressure $6 \mathrm{~Pa}$, discharge current $60 \mathrm{~mA}$ and the magnetic field strength $2.10^{-2} \mathrm{~T}$. The probe was positioned approximately in the middle between cathode and anode. The polar plots resemble circles even at lower energies what indicates almost isotropic EEFF. Qualitatively this is due to the effect of collisions of electrons with neutrals that randomise the directional movement of electrons caused by the magnetic field. When the pressure is lowered down to $0.71 \mathrm{~Pa}$ then at the same magnetic field $2.10^{-2} \mathrm{~T}$ and the same discharge current the picture changes. In Fig. 13 we present the numerically calculated second derivatives of the experimentally measured probe characteristics with respect to the probe voltage in three different angles to magnetic field. The curves taken at $0^{\circ}$ and $180^{\circ}$

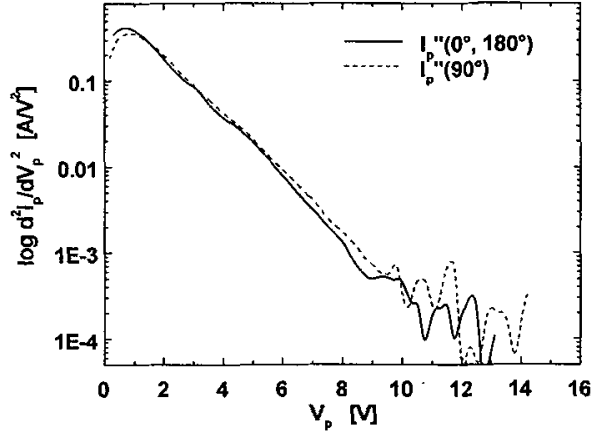

Figure 13. Second derivatives of the measured probe data in 3 different orientations at the conditions of Fig. 14.

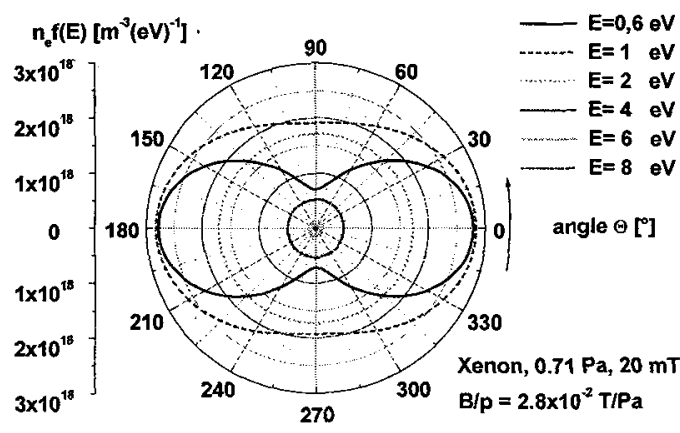

Figure 14. Polar plot of the EEDF in xenon at $0,71 \mathrm{~Pa}$ and $B=2.10^{-2} \mathrm{~T}$.

coincide. It is seen that at $90^{\circ}$ angle to $B$ the second derivative has smaller amplitude, its maximum is shifted towards higher energies and its tail decays slower with energy. This indicates higher mean electron energy in direction perpendicular to $B$. The polar plot in Fig. 14 shows already anisotropy of the EEDF that can be qualitatively described as deficit of the low energy electrons in direction perpendicular to $B$. This deficit is compensated by larger apparent temperature of electrons coming from this direction.

\section{CONCLUSION}

We presented our viewpoint on the problems that arise from the use of probe method in the transition regime of pressures and in a magnetic field. We used the method of comparison of the density values obtained from the electron and positive ion current part of the probe characteristic with certain success, 
however at higher pressures it is very difficult to fulfil the assumptions of the used theories. The presented method of the assessment of the anisotropy of EEDF in a magnetic field yields also the fairly simple method how to assure the quality of the experimental probe data under such conditions. In the presented contribution we concentrated on the measurement of the EEDF in non-isotropic plasma. The Mesentsev method [124] brings, however, also advantage when measuring the plasma density, since it solves by itself the problem of the reduction of the effective probe collecting area when the magnetic field strength is increased from $B=0$ (isotropic case) to highly magnetised plasma (non-isotropic case). The authors constructed a computer program under Microsoft Windows $\$$ that carries out the probe data processing in continuous interaction with the experimenter. The program is available, together with its fairly detailed description, on request on the e-mail of one of us (P.K.). In the future we would like to compare the presented theories with numerical simulations that are under way.

\section{Acknowledgements}

The work in Czech Republic was partially financially supported by the Grant Agency of Czech Republic, Grant No. GAČR-202/971011, by Grant Agency of Charles University, Grant No. GAUK-181/96 and by the EU Grant COPERNICUS CIPA-CT94-0183. The work in Germany was financially supported by the Deutsche Forschungsgemeinschaft (DFG) in frame of the project SFB 198 Greifswald "Kinetik partiell ionisierter Plasmen".

\section{References}

[1] Langmuir I., Mott-Smith H.M.,Gen. Elec. Rev. 26(1923)731.

[2] Langmuir I., Mott-Smith H.M., Gen. Elec. Rev. 27(1924)449, 538, 616, 762, 810.

[3] Mott-Smith, H.M., Langmuir I., Phys. Rev. 28(1926)727.

[4] Langmuir I., Phys. Rev. 28(1926) 104.

[5] Langmuir I., Phys. Rev. 31(1928)357.

[6] Godyak V.A., Piejak R.B., Alexandrovich B.M., Plasma Sources Sci. Technol. 1(1992)36.

[7] Waymouth J.F., Phys. Fluids 7(1964)1893; Phys. Fluids 9(1966)801.

[8] Swift J.D., Schwar M.J.R., Electrical Probes for Plasma Diagnostics (lliffe books, London, 1970).

[9] Waymouth J.F., J.Appl. Phys. 37(1966)4493.

[10] Godyak V.A., in Plasma-Surface Interaction and Processing of Materials, NATO ASI, Ser. E, vol. 176, O. Auciello et al., ed. (Kluwer Academic, Dordrecht, 1990) pp 95-134.

[11] Passoth E., Kudrna P., Csambal C., Behnke J.F., Tichý M., Helbig, V., J.Phys. D (Appl. Phys.) 30(1997)1763.

[12] Tichý M., Šícha M., David P., David T., Contrib. Plasma Phys. 34(1994)59.

[13] Tichý M., Š́cha M., David P., Proc. XX. ICPIG, Il Ciocco, Barga (Italy), July 8-12, 1991, V. Palleschi, M. Vaselli, Eds., Contributed Papers, p. 839.

[14] Chen F.F., in Plasma Diagnostics Techniques, ed. Huddelstone R.H.,Leonard S.L., (Academic Press, New York, London 1965).

[15] Granovskij V.L., Električeskij tok v gaze (GITTL, Moskva 1952), in russian, available also in german as Granowski; W.L., Der elektrische Strom im Gas (Akademie-Verlag, Berlin 1955).

[16] Chung P.M., Talbot L., Touryan K.J., Electrical Probes in Stationary and Flowing Plasmas, Theory and Application (Springer-Verlag, Berlin, Heidelberg, New York, 1975).

[17] Kapcov N. A., Elektronika (GITTL, Moskva 1953), in russian.

[18] Bohm D., Burhop E.H.S., Massey H.S.W., The Characteristics of Electrical Discharges in Magnetic Fields, A. Guthrie, R.K. Wakerling, Eds., (McGraw-Hill, New York 1949).

[19] Allen J. E., Boyd R.L.F., Reynolds., Proc. Phys. Soc. B70(1957)297.

[20] Chen F.F ., Plasma Phys. 7(1965)47.

[21] Bernstein I.B., Rabinowitz J.N., Phys. Fluids 2(1959)112.

[22] Laframboise J.G., Univ. of Toronto, Institute for Aerospace Studies (UTIAS), Report No. 100, (1966).

[23] Sonin A.A., ALAA Journal 4(1966)1588.

[24] Johnson E., Malter L., Phys. Rev. 80(1950)59.

[25] Hopkins M.B., Graham W.B., Rev.Sci.Instr. 57(1986)2210. 
[26] Smith D., Plumb I.C., J. Phys.D (Appl.Phys.) 6(1973)196.

[27] Klagge S., Tichý M., Czech. J. Phys. B 35(1985)988.

[28] Shulz G.J., Brown S. C., Phys Rev. 98(1955)1642.

[29] Tonks L., Langmuir I., Phys. Rev. 34(1929)876.

[30] Shaeffer J.F., PhD Thesis, University of St. Louis (1971).

[31] Virmont J., Proc. ICPIG -IX (Bucharest, Bulgaria 1969), Contrib. papers, p. 609.

[32] Trunec D., Španěl P., Smith D., Contrib. Plasma Phys. 35(1995)203.

[33] Goodall C.V., Smith D., Plasma Physics 10(1968)249.

[34] Goodall C.V., Polychronopoulos B., Planet. Space Sci. 22(1974)1585.

[35] Dunn M.G., Lordi J.A., AIAA Journal 7(1969)1458.

[36] Dunn M.G., Lordi J.A., ALAA Journal 8(1970)1077.

[37] Johnson R.A., DeBoer P.C.T., ALAA Journal 10(1972)664.

[38] Thornton J.A., ALAA Journal 9(1971)342.

[39] Zakharova V.M., Kagan J.M., Mustafin K.S., Perel V.I., Sov. Phys. Tech. Phys. 5(1960)411.

[40] Chen S-L, Goodings J.M., J. Appl. Phys. 39(1968)3300.

[41] Swift J.D., Proc. Phys. Soc. 79(1969)697.

[42] Waymouth J.F., Phys. Fluids 7(1964)1843.

[43] Talbot L., Chou Y. S., Rarefied Gas Dynamics (Academic Press, 1966) p. 1723.

[44] Chou Y. S., Talbot L., Willis D. R., Phys. Fluids $9(1966) 2150$.

[45] Kirchhoff R. H., Peterson E. W., Talbot L., AIAA Journ. 9(1971)1686.

[46] Bienkowski K.G., Chang K.W., Phys. Fluids 11(1968)784.

[47] Wasserstrom E., Su C.H., Probstein R.F., Phys. Fluids 8(1965)56.

[48] Self S.A., Shih C.H., Phys. Fluids 11(1968)1532.

[49] Shih C.H., Levi E., ALAA Journal 9(1971)1673, ALAA Journal 9(1971)2417.

[50] Klagge S., DSc Thesis (Greifswald University, 1988).

[51] Zakrzewski Z., Kopiczynski T., Plasma Physics 16(1974)1195.

[52] Kopiczynski T., PhD Thesis IMP PAN (Institute of Fluid-Flow Machines Polish Academy of Sciences, Gdansk, 1977).

[53] Basu J., Sen C., Japan J. Appl. Phys. 12(1973)1081.

[54] Schulz G. J., Brown S. C., Phys. Rev. 98(1955)1642.

[55] Jakubowski A. K., ALAA Journal 8(1972)988.

[56] David P., CSc Thesis (Faculty of Mathematics and Physics, Charles University, Prague 1985).

[57] David P., Šícha M., Tichý M., Kopiczynski T., Zakrzewski Z., Contrib. Plasma Phys. 30(1990)167.

[58] Kudrna, P., PhD Thesis, (Faculty Mathematics and Physics, Charles University, Prague 1996).

[59] Chudáček O., Kudrna P., Glosík J., Šícha M., Tichý M., Contrib. Plasma Phys. 35(1995)503.

[60] Šícha M., Zu Cham Sun, Glosík J., Tichý M., Contrib. Plasma Phys. 30(1990)195.

[61] Šícha M., Špatenka P., Tichý M., Contrib. Plasma Phys. 31(1991)43.

[62] Kagan J.M., Perel V.S., Uspekhi Fizicheskich Nauk 81(1963)409, in russian.

[63] Chang J.S., Laframboise J.G., Phys. Fluids 19(1976)25.

[64] Rogers J.H., DeGroot J.S., Hwang D.Q., Rev. Sci. Instr. 63(1992)31.

[65] Overzet L.J., Hopkins M.B., J. Appl. Phys. 74(1993)4323.

[66] . Sudit I.D., Woods R.D., J. Appl. Phys. 76(1994)4488.

[67] Johnsen R., Shun'ko E.V., Gougousi T., Golde M.F., Phys. Rev. E 50(1994)3994.

[68] Feld L., Litvinov I.I., Zhurnal Tekhnicheskoi fiziki 12(1968)1589, in russian.

[69] Sugawara M., Hatta Y., J. Appl. Phys. 36(1965)2361.

[70] Sugawara M., Phys. Fluids 9(1966)797.

[71] Litvinov I.I., Journal of Technical Physics 37(1967)2160, in russian.

[72] Kagan J.M., Perel V.I., Journal of Technical Physics USSR 38(1968)1663, (in russian).

[73] Litvinov I.I., Journal of Technical Physics USSR 40(1970)2018, in russian.

[74] Sanmartin J.R., Phys. Fluids 13(1970)103.

[75] Sato M., Phys. Fluids 15(1972)2427.

[76] Sato M., Phys. Fluids 17(1974)1903.

[77] Laframboise J.G., Rubinstein J., Phys. Fluids 19(1976)1900.

[78] Rubinstein J., Laframboise J.G., Phys. Fluids 21(1978) 1655.

[79] Rubinstein J., Laframboise J.G., Phys. Fluids 25(1982)1174. 
[80] Rubinstein J., Laframboise J.G., Phys. Fluids 26(1983)3624.

[81] Sonmor L.J., Laframboise J.G., Phys. Fluids 33(1991)2472.

[82] Rozhanski V.A., Tsendin L.D., Journal of Technical Physics USSR 48(1978)1647, in russian.

[83] . Vagner S.D., Shliaev B.V., Journal of Technical Physics USSR 48(1978)675, in russian.

[84] Vorobieva N.A., Zakharova V.M. Kagan J.M., Proceedings IX ICPIG, Bucharest (Bulgaria, 1969); p. 620.

[85] Dobe T., Amemiya H., J. Phys. Soc. Japan 19(1964)1915.

[86] Ichimiya M., Japan. Jour. Appl. Phys. 3(1964)789.

[87] Amemiya H., Dobe T., Japan. Jour. Appl. Phys. 7(1968)956.

[88] Amemiya H., Dobe T., Japan. Jour. Appl. Phys. 8(1969)818.

[89] Shilinsky A.P., Kubeiev B.V., Sacharov V.V., Smirnov A.S., Fizika plazmy 3(1977)1028, in russian.

[90] Demirchanov R.A., Leontiev N.J., Kosy J.A., Zhurnal tekhnicheskoi fiziki 32(1962)180, in russian.

[91] Jones H., Sanders P., J. Sci. Instr. 37(1960)457.

[92] Clements R.M., Skavsgard H.M., Can. J. Phys. 45(1967)3199.

[93] Ishi S., Miyoshi S., Okamoto K., Sakamoto Y., Proceedings IX ICPIG, Bucharest (Bulgaria, 1969), p. 563.

[94] Ishi S., Miyoshi S., Japan. J. Appl. Phys. 19(1980)719.

[95] Gyergyek T., Čerček M., Jelić N., Stanojević M., Contrib. Plasma Phys. 33(1993)53.

[96] Stanojević M., Čerček M., Gyergyek T., Jelić N., Contrib. Plasma Phys. 33(1993)241.

[97] Stanojević M., Čerček M., Gyergyek T., Jelić N., Contrib. Plasma Phys. 34(1994)607.

[98] Hansen K., Klinger T., Piel A., Rev. Sci. Instr. 65(1994)2615.

[99] Špatenka P., Leipner I., Vlček J., Musil J., Plasma Sources Sci. Technol. 6(1997)1.

[100] Kudrna P., Passoth E., Contrib. Plasma Phys. 37(1997) in print.

[101] Bertotty B., Phys. Fluids 4(1961)1010.

[102] Bertotty B., Phys. Fluids 4(1961)1047.

[103] Peter G., Rybarsch H., Beitr. Plasmaphys. 16(1976)333.

[104] Stangeby P.C., J.Phys. D Appl. Phys. 20(1987)1472.

[105] Stangeby P.C., in Physics of plasma-wall interaction in controlled fusion, D.E. Post, R. Behrsch, Eds. (Plenum publishing corporation, 1986).

[106] Stangeby P.C., J.Phys. B 15(1982)1007.

[107] Stangeby P.C., Phys. Fluids 27(1984)682.

[108] Brown J.G., Compher A.B., Kunkel W.B., Phys Fluids 14(1971)1377.

[109] Cohen I.M., Phys. Fluids 12(1969)2356.

[110] Lam S.H., Phys. Fluids 8(1965)1002.

[111] Ditte U., Müller K.G., Proceedings XVI. ICPIG, Contributed Papers, Düsseldorf (Germany, 1983), p. 124.

[112] Blancod J.C., Golovanievski K.S.,Kravchov T.P., Proceedings X. ICPIG, Oxford (UK, 1971) p. 25.

[113] Aikawa H., J, Phys. Soc. Japan 40, 1741 (1976).

[114] Brown J.G., Compher A.B., Ehlers K.W., Hopkins D.F., Kunkel W.B., Rostler P.S., Plasma Phys, 13(1971)47.

[115] Zakrzewski Z., Kopiczynski T., Lubanski M., Czech.J.Phys. B 30(1980)1167.

[116] Arslanbekov R.R., Khromov N.A., Kudryavtsev A.A., Plasma Sources Sci. Technol. 3(1994)528.

[117] Godyak V.A., Piejak R.B., Alexandrovich B.M., J. Appl. Phys. 73(1993)3657.

[118] Fernández Palop J.I., Ballessteros J., Colomer V., Hernández M.A., Rev. Sci. Instr. 66(1995)1.

[119] Mesentsev A.P., Mustafaev A.S., Federov V.L., J.Tech.Phys. USSR (Sov.Phys. Tech.Phys) 55(1985)544, in russian.

[120] Mesentsev A.P., Mustafaev A.S., Federov V.L., J.Tech.Phys. USSR (Sov.Phys. Tech.Phys.) 55(1985)2232, in russian.

[121] Mesentsev A.P., Mustafaev A.S., Federov V.L., J.Phys.D 21(1988)1464.

[122] Federov V.L., J.Tech.Phys. USSR (Sov.Phys. Tech.Phys) 55(1985)926, in russian.

[123] Laphshin V.F., Mesentsev A.P., Mustafaev A.S., Federov V.L., Proceedings ESCAMPIG-IX, C.M. Ferreira, Ed., Lisabon, (Portugal, 1988), p 141.

[124] Mesentsev A.P., Mustafaev A.S., Laphshin V.F., Federov V.L., J.Phys.B 20(1987)L723, J.Phys.B 20(1987)L923.

[125] Laphshin V.F., Mustafaev A.S., J.Tech.Phys.USSR (Sov.Phys. Tech.Phys) 59(1989)35.

[126] Federov V.L., Mesentsev A.P., J.Tech.Phys.USSR (Sov.Phys. Tech.Phys) 57(1987)595.

[127] Klagge S., Lunk A., J. Appl.Phys. $70(1991) 99$. 